\title{
Should early enteral nutrition be used in the trauma intensive care unit?
}

Critical illness, sepsis, surgery, multi-organ failure and haemodynamic instability are conditions that are associated with feeding inadequacies and nutritional challenges. Conversely, meeting nutritional requirements and providing optimal nutrition are associated with an improved outcome. Since these facts have been reported repeatedly, why then are we still faced with study results on suboptimal feeding?

The majority of clinical practice recommendations refer to medical and surgical intensive care unit (ICU) patient categories, with differences in their approach. However, recommendations on feeding critically ill trauma patients are scanty. Increased metabolism during the acute phase period, which can persist for as long as a year in severely burnt patients, for instance, is a characteristic feature of trauma patients. ${ }^{1}$ This period is also characterised by severe protein catabolism, which, in turn, necessitates increased protein intake to compensate for nutrient losses. ${ }^{2}$ A protein intake of $\geq 2 \mathrm{~g} / \mathrm{kg} /$ day was needed to ensure a protein balance in a recent study on trauma patients. $^{3}$

Even though nutrition experts may not necessarily agree on the optimal timing of the initiation of parenteral nutrition, all agree that the enteral route should be used as the first option, provided that the gut is functional. ${ }^{2,4-8}$ The concept of early enteral feeding (EEF) refers to the initiation of enteral nutrition within the first 24-48 hours post injury. ${ }^{4-9}$ Advantages of this approach include a reduction in infectious complications and mortality. ${ }^{4,-9}$ Various additional benefits of EEF have also been reported, including the fact that it lends support to the gastrointestinal tract responses by maintaining gut integrity and improving absorptive capacity, to the immunological profile by modulating the immune response to enhance the systemic immune function, as well as to the associated metabolic responses by improving insulin sensitivity to aid with glucose control. ${ }^{9}$

The earlier enteral feeding can be resumed post injury, the quicker optimal nutritional requirements can be met. The latter refers to both energy and protein requirements. By keeping energy and protein deficits to a minimum, complications associated with the said deficits can be minimised, i.e. renal impairment, pressure sores, additional surgery needs, prolonged mechanical ventilation, extended length of stay, nosocomial infections and increased mortality. ${ }^{1-5,8}$ The benefits and outcomes of EEF in critically ill trauma patients were investigated in a local study, the first large study in this population in South Africa. It is reported by Löfgren et al in this issue of the SAJCN. ${ }^{10}$ EEF was associated with a significantly reduced length of stay and mortality. The level of significance of these findings was different across the different injury severity scores used. The complications relating to EEF and the achievement of caloric goals were also assessed in another study by the same group, as reported by Aaben et al, ${ }^{11}$ also in the current $S A J C N$ issue. The late attainment of enteral goal feeding was associated with a hazard ratio of 2.67 for the development of sepsis, compared to that associated with achieving the feeding goals early. Similarly, the late initiation of enteral feeding was associated with a hazard ratio of 2.41 for an increased sepsis rate. These findings are in line with international experience, afford local perspective and support the concept that the early initiation of enteral feeding, coupled with the early attainment of feeding goals, is associated with an improved outcome.

The implementation of a feeding protocol in ICUs as a means of ensuring that optimal nutritional needs are met as early as possible ${ }^{4}$ has received much attention and remains a point of vigorous debate. The purpose of the said protocols are to ensure that healthcare professionals dealing with patients at all times of day or night have guidance on how to optimally build the nutritional intake of patients or deal with any nutritional intolerance. Thus, rather than stopping feeds, alternative options, such as changing the hourly feeding rate, changing the formula or changing the feeding route (including combined route options), should be the priority approach.

Two newer versions of the traditional protocols have recently been developed and tested. These are the enhanced protein-energy provision via the enteral route in critically ill patients (PEP uP $)^{12}$ and the Feed Early Enteral Diet adequately for Maximum Effect (FEED $\mathrm{ME})^{13}$ protocols. Volume-based goals drive the new aggressive approaches. Therefore, instead of starting slowly and increasing slowly until the goals are reached, this approach determines the hourly rate based on the 24-hour volume goal. If the patient falls behind, the hourly rate is adjusted to ensure that the total intake is met within a given 24-hour period. ${ }^{12,13}$

Successful implementation of the PEP uP protocol in mechanically ventilated ICU patients resulted in significantly more energy and protein delivered per 24 hours. No differences in gastrointestinal side-effects were experienced when compared to the traditional approach. ${ }^{12}$ The FEED ME protocol was also tested on mechanically ventilated patients in a surgical trauma ICU. Significantly more energy and protein intake was also reported, with similar episodes of emesis and raised gastric residual volumes between the groups. ${ }^{13}$ One of the differences between the PEP up and FEED ME protocols is that FEED ME is implemented once patients have reached the goal rate of enteral nutrition, as opposed to the PEP UP protocol which entails feeding immediately that the enteral route can be used. A make-up component is calculated in both protocols to ensure that full daily needs are met. This is achieved by adjusting the enteral nutrition prescribed goal rate in FEED ME versus adjusting the hourly 
rate to ensure that the prescribed enteral nutrition total daily volume is reached in PEP uP. However, both protocols seem to be successful in ensuring a better intake without sacrificing gastrointestinal tolerance. ${ }^{12,13}$

A few general feeding recommendations which can be applied to critically ill trauma patients include: ${ }^{1-3,5-7}$

- Starting enteral nutrition as soon as possible after ICU admission, increasing it according to gut tolerance, and administering prokinetics, if needed.

- Reassessing gut function and feeding tolerance daily, and making adjustments accordingly.

- Ensuring an energy intake of $20-25 \mathrm{kcal} / \mathrm{kg} /$ day during the early acute phase, and $25-30 \mathrm{kcal} / \mathrm{kg} /$ day during the stabilised phase. Alternatively, $120-140 \%$ of basal energy expenditure can be used.

- Ensuring a protein intake of $1.5-2.0 \mathrm{~g} / \mathrm{kg} /$ day, maintained through all of the phases.

- Avoiding overfeeding.

- Being aware that glucose control is important.

- Adding glutamine to all enteral formula in polytrauma patients.

- Employing a protocol-based approach to ensure that optimal intake is reached.

Trauma patients experience raised nutritional needs. Meeting patients' requirements in the quickest possible manner through the implementation of early enteral nutrition is associated with an improved outcome in terms of morbidity and mortality, and should be strived for at all times.

\section{Reneé Blaauw}

Division of Human Nutrition, Stellenbosch University

\section{References}

1. Wade $\mathrm{CE}, \mathrm{Kozar} \mathrm{RA}$, Dyer $\mathrm{CB}$, et al. Evaluation of nutrition deficits in adult and elderly trauma patients. JPEN Parenter Enteral Nutr. 2015;39(4):449-455.

2. Berette L, Rocchetti S, Braga M. What's new in emergencies, trauma and shock? Nitrogen balance in critical patients on enteral nutrition. J Emerg Trauma Shock. 2010;3(2):105-108.

3. Dickerson RN, Pitts SL, Maish G0, et al. A reappraisal of nitrogen requirements for patients with critical illness and trauma. J Trauma Acute Care Surg. 2012;73(5):549-557.

4. McClave SA, Heyland DK. The physiologic response and associated clinical benefits from provision of early enteral nutrition. Nutr Clin Pract. 2009;24(3):305-315.

5. Singer P, Hiesmayr M, Biolo G, et al. Pragmatic approach to nutrition in the ICU: expert opinion regarding which calorie protein target. Clin Nutr. 2014;33(2):246-251.

6. Malpica ALB, Mateos AGDL, Gonzalez AR. Guidelines for specialized nutritional and metabolic support in the critically-ill patient: update. Consensus SEMICYUC-SENPE: multiple trauma patient. Nutr Hosp. 2011:26 Suppl 2:63-66.

7. Doig GS, Heighes PT, Simpson F, Sweetman EA. Early enteral nutrition reduces mortality in trauma patients requiring intensive care: a meta-analysis of randomised controlled trials. Injury. 2011;42(1):50-56.

8. Elke G, Wang M, Weiler N, et al. Close to recommended caloric and protein intake by enteral nutrition is associated with better clinical outcome of critically ill septic patients: secondary analysis of a large international nutrition database. Crit Care. 2014;18(1):R29.

9. Martindale RG, Warren M. Should enteral nutrition be started in the first week of critical illness? Curr Opin Clin Nutr Metab Care. 2015;18(2):202-206.

10. Löfgren E, Mabesa T, Hammarqvist F, Hardcastle TC. Early enteral nutrition compared to outcome in critically ill trauma patients at a level one trauma centre. South Afr Clin J Nutr. 2015;28(2):xx-xx.

11. Aaben C, Hammarqvist F, Mabesa T, Hardcastle TC. Complications relating to enteral and parenteral nutrition in trauma patients: a retrospective study at a level one trauma centre in South Africa. South Afr Clin J Nutr. 2015;28(2):62-67.

12. Heyland DK, Cahill NE, Dhaliwal R, et al. Enhanced protein-energy provision via the enteral route in critically ill patients: a single center feasibility trial of the PEP UP protocol. Crit Care. 2010;14(2):R78.

13. Taylor B, Brody R, Denmark R, et al. Improving enteral delivery through the adoption of the Feed Early Enteral Diet Adequately for Maximum Effect (FEED ME) protocol in a surgical trauma ICU: a quality improvement review. Nutr Clin Pract. 2014;29(5):639-648. 